\title{
Recherche de propriétés intrinsèques du bois pouvant expliquer la sensibilité à la gélivure de Quercus petraea (Liebl) et $Q$ robur (L)
}

\author{
B Cinotti * \\ École nationale du Génie Rural, des Eaux et des Forêts, laboratoire de recherche en sciences \\ forestières 14, rue Girardet, 54042 Nancy Cedex; INRA, station de recherches \\ sur la qualité des bois, centre de Nancy, 54280 Champenoux, France
}

(Reçu le 22 février 1990; accepté le 9 avril 1991)

Résumé - la comparaison, au sein de populations de chênes du Centre et de l'Est de la France, des arbres ayant gélivé et des arbres ayant résisté au froid a établi la grande variabilité de la sensibilité à la gélivure et l'existence de facteurs individuels prédisposants (densité, humidité hivernale, angle de fil du bois, retraits, nombre de rayons ligneux, surface des vaisseaux du bois initial, largeur de cerne). Cette façon de qualifier le bois des arbres ayant gélivé a apporté des compléments d'information à toutes les investigations récentes sur les facteurs prédisposants stationnels et sylvicoles. Toutefois, certains éléments anatomiques qui semblent impliqués dans le phénomène, tels que les rayons ligneux, n'ont pas pu être étudiés avec tout le détail nécessaire et souhaité.

bois / gélivure / variabilité / anatomie / Quercus

Summary - Investigation of the intrinsic properties of wood for explaining the occurrence of frost-crack in Quercus petraea (Liebl) and $Q$ robur (L). Comparison, within oak stands from central and eastern parts of France, of frost-cracked and sound trees has established the great variability of sensitivity to frost-crack and the existence of individual predisposing factors (specific gravity, winter moisture content, grain angle, shrinkage, number of rays, early wood vessel cross sectional area and ring width). These methods of characterizing the wood of frost-cracked trees have brought complementary information to all recent research about site and silvicultural predisposing factors. Nevertheless, some anatomical features, supposedly linked with frost-crack occurrence, such as rays, have not been studied in a sufficiently detailed way.

wood / frost / crack / variability / anatomy / Quercus

\footnotetext{
* Adresse actuelle : ministère de l'Agriculture et de la Forêt, service central des enquêtes et études
} statistiques, 4, avenue de St-Mandé, 75570 Paris Cedex 12 


\section{INTRODUCTION}

La caractérisation des paramètres individuels prédisposant à la gélivure a été une des démarches menées au cours d'un travail de thèse auquel le lecteur pourra se reporter (Cinotti, 1989). Elle a notamment consisté à rechercher si, de la très grande variabilité intraparcellaire de la sensibilité des chênes à la gélivure, il ressort différentes caractéristiques individuelles (dendrométrie, densité, retraits du bois, angle de fil, largeur de cerne ou anatomie) de leur bois qui qualifient les arbres sensibles à la gélivure, par comparaison aux arbres ayant résisté. Une étude similaire a déjà été menée, pour l'anatomie, par Savill (1986), pour caractériser la propension des chênes à la fente.

Si certaines des caractéristiques du bois présentent des valeurs différentes dans les deux groupes, il est possible qu'on ait mis le doigt sur des facteurs prédisposants individuels ou en liaison avec des facteurs prédisposants stationnels ou sylvicoles. L'intérêt pour l'améliorateur de tels caractères différenciant les arbres sensibles des autres est évident.

\section{MATÉRIELS ET MÉTHODES}

\section{Matériel végétal étudié}

Une première série de 34 arbres, qui seront dénommés "arbres 1987", a été prélevée dans deux forêts domaniales, Allogny (Cher) et Languimberg (Moselle). Les arbres provenant d'Allogny étaient 26 chênes sessiles (Quercus petraea Liebi), ceux du Languimberg huit pédonculés ( $Q$ robur $L)$. lls ont été choisis deux par deux, un gélivé, un témoin, proches l'un de l'autre, de même diamètre à $1,30 \mathrm{~m}$ et de même forme générale de façon à neutraliser les effets de sol, de diamètre et de forme.

Une deuxième série d'arbres qui seront dénommés "arbres 1988" a été prélevée, suivant les mêmes protocoles que ceux de 1987, dans trois autres forêts, forêts domaniales de Bellary (Nièvre) et de Vierzon (Cher) dans le Centre, et forêt domaniale de Mouterhouse (Moselle) dans l'Est. Les 30 arbres provenant de Bellary et les 34 de Mouterhouse étaient des chênes sessiles, les 30 de Vierzon des pédonculés. Dans la suite de cet article la forêt de Mouterhouse sera désigné par la région naturelle à laquelle elle appartient : Bitche.

\section{Prélèvements et mesures réalisés}

Chaque arbre est représenté par une rondelle prélevée à plus de $50 \mathrm{~cm}$ de la découpe basale afin d'éviter les modifications de structure du bois au voisinage de l'empattement. Chaque rondelle a été découpée suivant un schéma présenté dans les pages qui suivent. Toutefois, pour les arbres de 1987 et pour les mesures d'angle de fil, chaque rayon a été divisé, non pas en trois (aubier, duramen, cœur), mais en dix zones d'égal nombre de cernes allant de la moelle à l'écorce.

La densité étudiée ici est l'infradensité, rapport de la masse anhydre au volume saturé. Pour les arbres de 1987, la différence de densité entre le petit côté (axe du plus petit rayon) et le côté qui lui faisait face a aussi été étudiée.

Les retraits ont été mesurés entre l'état saturé et l'état sec à l'air. L'humidité exacte des échantillons ayant servi à cette mesure, contrôlée sur une série de témoins par deux pesées : une à l'état "sec à l'air», une à l'état anhydre, était de $14,6 \%$.

L'évaluation du retrait sur les arbres 1987 a été effectuée au coordinatographe RolletVendôme par mesure optique de la déformation. Cette méthodologie s'est révélée d'une précision trop faible pour que les mesures soient répétables, ce qui a donné de très mauvais résultats sur certaines zones dont les limites étaient mal définies. L'ensemble des résultats pour les variables "Retrait radial" et "Anisotropie de retrait» pour les arbres 1987 ne seront donc pas présentés. En ce qui concerne les arbres de 1988, la mesure a été faite au pied à coulisse.

La mesure de l'angle de fil du bois par la méthode de fendage d'une rondelle et l'observation des deux profils radiaux résultants sont controversées. La précision du fendage et les effets 




Fig 1. Plan de découpe des billons.

possibles de déviation du fil par des nœuds non apparents sur la qualité de la mesure ne sont pas irréprochables. Malheureusement, la découpe d'un seul disque par arbre ne permet pas d'avoir une idée de l'erreur commise sur ces mesures. Cette mesure a malgré tout été menée à son terme puisque les tentatives de 1987 avaient été couronnées de succès d'une part, et que, d'autre part, l'angle de fil est un élément d'aggravation des contraintes internes des tiges (Ferrand, 1982). Nous ne pouvions donc négliger ce caractère.

L'humidité hivernale des chênes a été étudiée sur des carottes diamétrales prélevées à la tarière dans des couples de chênes gélivé-sain, toujours suivant le même azimut. Ces carottages ont été effectués à Bellary en janvier, février et mars, Mouterhouse en mars, et Vierzon 
en février et mars. Dès le prélèvement, nous avons placé les carottes dans des tubes de plastique de diamètre intérieur égal à celui des carottes et bouchés à chaque extrémité pour limiter au maximum les échanges d'eau. La perte d'humidité des carottes dans les tubes peut être estimée à $0,2 \%$ (en valeur absolue). Ce biais systématique (perte d'eau des échantillons les plus humides, peut-être partiellement compensée par une reprise d'humidité des plus secs placés dans le même tube) est difficile à corriger mais l'effet de lissage des humidités extrêmes semble - au vu des résultats qui suivent - avoir été réduit. Les carottes ont ensuite été coupées en six morceaux (aubier, duramen, cœur, côtés Nord et Sud), pesées à l'état humide, séchées en étuve à $103^{\circ} \mathrm{C}$ pendant une journée et enfin pesées à l'état anhydre.

Les mesures anatomiques quantitatives ont été réalisées sur des clichés radiographiques à l'aide de l'analyseur d'image dont dispose la station de recherches sur la qualité des bois, sauf celles concernant les gros rayons ligneux qui ont été comptées à l'œil. Nous précisons à toutes fins utiles que par "gros rayons ligneux" nous entendons les rayons plurisériés, c'est-àdire constitués de plusieurs files tangentielles de cellules.

En effet, ne pouvant procéder, avec cet analyseur d'images, à la mesure de la fraction surfacique du bois en rayons ligneux (ni a fortiori à aucune autre mesure élaborée de topologie de ces rayons), nous nous sommes limités à une méthode de comptage manuel de ces gros rayons, sur une corde de $3,5 \mathrm{~cm}$ de longueur, sur deux planches radiales diamétralement opposées des arbres 1988.

Pour la première mesure anatomique sur 14 échantillons (ie sept couples, cinq de la forêt d'Allogny, deux de celle du Languimberg) de la série 1987 à l'aide de l'analyseur d'images, chaque rayon avait été divisé, du cœur à l'écorce, en 10 zones d'égal nombre de cernes. La mesure n'a toutefois porté que sur la troisième zone en partant de la moelle (située selon les arbres, et leur âge, entre le $15^{\circ}$ et le $25^{\circ}$ cerne) (Cinotti, 1987). Cette mesure a été répétée sur l'ensemble des 10 zones de 16 échantillons (huit couples, cinq d'Allogny, trois du Languimberg) de la même série par Haag (1989) et accompagnée alors d'une étude de répétabilité des mesures.

\section{Méthodes d'analyse des données}

Sur la série d'arbres de la campagne 1987, les mesures (sauf les mesures anatomiques à l'analyseur d'image) ont été traitées en analyse de variance à deux facteurs :

- facteur "couple» (13 couples pour Allogny, 4 pour Languimberg);

- facteur "gélivure" (gélivé, témoin); pour les analyseurs au niveau arbre (dendrométrie et anatomie).

Un troisième facteur a été ajouté pour les analyses détaillées zone par zone (angle de fil, densité, retraits, largeur moyenne de cerne) :

- le facteur "zone».

Les données de la série "arbres 1988" ont été traitées, forêt par forêt, en analyse de variance à quatre facteurs, sauf cas particulier des caractères dendrométriques, de l'angle de fil du bois, et du nombre des rayons lingeux :

- "couple" (15 couples d'arbres pour Bellary, 17 pour Bitche, 15 pour Vierzon);

- "gélivure" (gélivé, témoin);

- "position sur le rayon" (15 premiers cernes en partant de la moelle dénommés "cœur", le reste du duramen dénommé “duramen", aubier);

- "opposition diamétrale" (petit côté et celui qui lui fait face), pour faire apparaître d'éventuels effets de bois de réaction, sauf pour l'humidité (pour laquelle il s'agit de l'opposition Nord-Sud entre les deux prélèvements de carottes).

Les données résultant du comptage des gros rayons ligneux des arbres de 1988 ont été analysées en analyse de variance à trois facteurs :

- couple (47 couples);

- gélivure;

- opposition diamétrale.

Les données dendrométriques et d'angles de fil ont été analysées en analyse de variance à trois facteurs :

- couple (47 couples);

- gélivure;

- zone.

Les tableaux de comparaison de moyenne des pages qui suivent présentent les moyennes (générales, gélivés et témoins) ainsi que le ré- 
sultat de la comparaison des moyennes issu de l'analyse de variance. Les effets position et opposition ne sont pris en considération que pour leurs interactions avec le facteur gélivure. Sur ces tableaux, la présence d'une, deux ou trois astérisques ou de l'abréviation NS, signifie que les résultats sont respectivement significativement différents entre les gélivés et les nongélivés au seuils de $5,1,0,1 \%$ ou qu'ils ne sont pas significativement différents au seuil de $5 \%$.

\section{RÉSULTATS}

\section{Caractéristiques dendrométriques et sensibilité à la gélivure}

Notre étude des facteurs individuels prédisposants était dès l'origine limitée aux paramètres descriptifs du bois. La récolte des données dendrométriques n'avait pour but que de permettre un contrôle sur l'identité d'âge et de diamètre moyen des deux populations comparée; ceci, afin de pallier l'influence de la forme de l'arbre et de sa position sociale dans le peuplement sur sa sensibilité à la gélivure, que les travaux de Miller (1987) et de l'IDF (1988) laissent supposer.

\section{Arbres 1987 (forêts d'Allogny et du Languimberg)}

La circonférence à mi-hauteur totale de bille est, de toutes les variables analysées (hauteur totale, hauteur sous houppier, circonférence à mi-hauteur totale, diamètres croisés du houppier, nombre de cernes à la souche), la seule significativement différente entre gélivés et non-gélivés (elle est plus grande pour les gélivés qui, à diamètre à 1,30 m égal, rappelons-le, seraient donc plus cylindriques). Les autres caractères dendrométriques relevés en 1987 ne diffèrent pas de façon significative. Ce résultat ne paraît pas pouvoir susciter d'interprétation particulière, car la circonférence à mi-hauteur n'est pas une caractéristique dendrométrique fréquemment étudiée. Elle n'avait été notée que pour permettre, le cas échéant, le calcul d'un volume de tige.

\section{Arbres 1988}

Les chênes sessiles de 1988 gélivés et témoins ne diffèrent ni par l'âge, ni par le rayon, ni par la largeur moyenne de cerne, ce qui semble cohérent. Notre échantillonnage n'est pas biaisé de ce point de vue, ce qui est important puisque certains auteurs signalent une influence du diamètre de l'arbre sur sa sensibilité à la gélivure.

L'épaisseur de l'écorce est supérieure pour les arbres gélivés à celle des arbres témoins. II était pourtant imaginable qu'une écorce plus épaisse constituât un frein aux échanges thermiques entre extérieur et intérieur. II semble donc probable que ces échanges dépendent aussi du nombre de fissures existant dans l'écorce, ou de sa conformation (ce dont les mesures effectuées ne peuvent rendre compte) et que l'épaisseur de l'écorce ne constitue pas à elle seule un bon indicateur de la protection thermique.

D'Arbois de Jubainville (1878) pensait que, l'aubier étant plus humide, les tiges qui en comporteraient une plus grande proportion seraient plus sensibles au froid. L'absence de différence de largeur et de proportion d'aubier par rapport au rayon entre arbres gélivés et témoins n'apporte pas d'éléments de vérification de cette hypothèse. La différence de nombre de cernes d'aubier entre arbres gélivés et témoins implique, quant à elle, une différence de largeur de cernes dans l'aubier, puisque la largeur d'aubier ne diffère pas. La largeur moyenne des cernes sur l'ensemble du rayon étant la même pour les arbres gélivés et les témoins $(1,74 \mathrm{~mm}$; voir tableau I), nous pouvons ajouter que les arbres gélivés accusent un ralentissement de croissance au cours des 20 der- 
Tableau I. Caractéristiques dendrométriques de chênes sessiles de 1988 (forêt de Bellary et de Bitche).

\begin{tabular}{lccccc}
\hline & Moyenne & Gélivés & & Témoins \\
& & & & & \\
\hline Âge de l'arbre & 98 & 98 & $=$ & 98 & NS \\
Rayon de l'arbre (en mm) & 162 & 163 & $=$ & 160 & NS \\
Largeur de cerne (en mm) & 1,74 & 1,74 & $=$ & 1,74 & NS \\
Épaisseur de l'écorce (en mm) & 7,7 & 8,2 & $>$ & 7,2 & $*$ \\
Largeur de l'aubier (en mm) & 27 & 27 & $=$ & 27 & NS \\
Proportion (en \% du rayon) & 17 & 17 & $=$ & 18 & NS \\
Nombre de cernes d'aubier & 19 & 21 & $>$ & 18 & $*$ \\
& & & & & \\
\hline
\end{tabular}

nières années plus important que les arbres témoins $(27 \mathrm{~mm} / 21$ ans contre $27 \mathrm{~mm} / 18$ ans).

L'analyse des mêmes caractéristiques dendrométriques conduite sur les chênes pédonculés de la forêt de Vierzon n'a mis en évidence aucune différence entre arbres gélivés et arbres témoins, ainsi que l'indique le tableau II.

\section{Conclusion}

\section{sur les caractères dendrométriques}

Comme cela a déjà été signalé, l'objet de cette étude n'était pas l'examen des caractères dendrométriques, mais la vérification de la similitude des groupes d'arbres gélivés et témoins. C'est chose faite.

Par ailleurs, ces résultats sur les caractères dendrométriques ne contredisent en rien les travaux de Miller (1987) et de l'IDF (1988) qui laissent penser qu'il existe une influence de la forme de l'arbre (inclinaison, forme de la tige et du houppier) et de sa position sociale dans le peuplement, sur sa sensibilité à la gélivure.

\section{Densité du bois et sensibilité à la gélivure}

L'influence de la densité sur les propriétés mécaniques du bois et sur sa rétractibilité

Tableau II. Caractéristiques dendrométriques des chênes pédonculés de 1988 (forêt de Vierzon).

\begin{tabular}{lccccc}
\hline & Moyenne & Gélivés & & Témoins & \\
\hline & & & & & \\
Âge de l'arbre & 85 & 81 & $=$ & 90 & NS \\
Rayon de l'arbre (en mm) & 117 & 118 & $=$ & 116 & NS \\
Largeur de cerne (en mm) & 1,78 & 1,70 & $=$ & 1,85 & NS \\
Eppaisseur de l'écorce (en mm) & 10,3 & 9,7 & $=$ & 10,8 & NS \\
Largeur de l'aubier (en mm) & 25 & 26 & $=$ & 23 & NS \\
Proportion (en \% du rayon) & 21 & 22 & $=$ & 20 & NS \\
Nombre de cernes de d'aubier & 19 & 19 & $=$ & 20 & NS \\
\hline
\end{tabular}


est bien connue. C'est pourquoi nous avons cherché d'éventuelles relations entre ce caractère et la sensibilité individuelle à la gélivure.

\section{Arbres 1987}

L'étude menée sur les arbres de 1987, sur deux rayons diamétralement opposés, avait pour but de faire apparaître des différences éventuelles d'infradensité entre arbres gélivés et arbres restés témoins, ou d'un côté de l'arbre à l'autre. Ces infradensités, présentées dans le tableau III, sont significativement supérieures pour les arbres gélivés, d'un côté comme de l'autre. En outre, il n'y a pas d'interaction entre le facteur gélivure et le facteur zone. Enfin, la différence d'infradensité entre les deux côtés, qui aurait pu indiquer la présence de bois de réaction, n'est jamais significative.

\section{Arbres 1988}

Alors que les infradensités des arbres gélivés et témoins (tableau IV) ne diffèrent pas dans les forêts de Bellary et de Bitche (chêne sessile), elles diffèrent significativement au seuil de $5 \%$ à Vierzon (chêne pédonculé) : le bois des arbres gélivés y est généralement plus dense que celui des arbres demeurés indemnes. La discrimination observée sur les arbres de 1987 n'apparaît donc pas dans toutes les forêts.

Tableau III. Infradensités des chênes de 1987; sessiles et pédonculés mélangés (en $\mathrm{kg} / \mathrm{m}^{3}$ ).

Moyenne Gélivés Témoins

$\begin{array}{lll}\text { Rayon petit côté } & 540 & 546>534 \\ \text { Rayon côté opposé } & 554 & 563>545^{* * *}\end{array}$

Tableau IV. Infradensités des chênes de 1988 (en $\mathrm{kg} / \mathrm{m}^{3}$ ) pour chacune des trois forêts étudiées.

\section{Moyenne Gélivés Témoins}

\begin{tabular}{lllll}
\hline Bellary & 569 & 567 & $=571$ NS \\
Bitche & 550 & 552 & $=547$ NS \\
Vierzon & 537 & $541>534 *^{*}$ \\
\hline
\end{tabular}

L'absence d'effet "gélivure" dans les forêts de Bellary et de Bitche est toutefois tempérée par le caractère significatif des interactions couple-gélivure et positiongélivure, ce qui signifie que pour certains couples ou dans certaines positions sur le rayon (tableau $\mathrm{V}$ ), des différences significatives peuvent exister.

\section{Conclusion sur l'infradensité}

Sur l'ensemble de nos dispositifs, les infradensités des populations gélivée et témoin ne sont pas toujours significativement différentes, mais quand elles le sont, c'est en général l'infradensité des arbres gélivés qui est la plus forte (en général, car l'aubier des arbres de Bitche fait exception à cette règle). Cette augmentation de densité devrait en principe induire une augmentation de la rigidité. Nos observations viennent étayer la remarque de d'Arbois de Jubainville (1878) : «les chênes à bois dense et nerveux sont plus exposés à la gélivure".

\section{Retraits du bois et sensibilité à la gélivure}

\section{Retrait longitudinal des arbres de 1987}

Malgré un coefficient de variation résiduel de $22 \%$ qui permet de penser que les fac- 
Tableau V. Interaction gélivure-position sur l'infradensité (en $\mathrm{kg} / \mathrm{m}^{3}$ ).

\begin{tabular}{|c|c|c|c|c|c|c|c|c|c|}
\hline & & & & Dur & men & & $A u$ & & \\
\hline & gélivés & témoins & gélivés & & témoins & gélivés & & témoins & \\
\hline Bellary & $591=$ & 584 & 585 & $=$ & 588 & 538 & $>$ & 525 & * \\
\hline Bitche & $608>$ & 591 & 564 & $>$ & 551 & 483 & $<$ & 499 & $* * *$ \\
\hline
\end{tabular}

teurs de l'analyse décrivent assez bien la variabilité du retrait longitudinal, aucune différence n'est apparue entre arbres gélivés et témoins en 1987 (tableau VI). Ce caractère n'a pas été mesuré sur les arbres 1989).

\section{Retrait tangentiel}

Le retrait tangentiel (tableau VII) des arbres gélivés de 1987 et de ceux de Bellary est significativement supérieur à celui des arbres demeurés indemnes; aucune

Tableau VI. Retrait longitudinal des chênes de 1987 (en \%).

Moyenne Gélivés Témoins

$\begin{array}{lll}\text { Moyennes } & 0,68 & 0,69=0,68 \quad \text { NS }\end{array}$
arbres 1987

Tableau VII. Retraits tangentiels des chênes de 1987 et 1988 (en \%).

\section{Moyenne Gélivés Témoins}

$\begin{array}{llllll}\text { Arbres } 87 & 8,27 & 8,47 & > & 8,06 & * * * \\ \text { Bellary } & 6,99 & 7,14 & > & 6,83 & * * \\ \text { Bitche } & 9,05 & 9,09= & 9,01 & \text { NS } \\ \text { Vierzon } & 7,94 & 7,72< & 8,12 & * * *\end{array}$

différence n'apparaît à Bitche. À Vierzon, le retrait est supérieur pour les arbres témoins, ce qui va à l'encontre des résultats de 1987 et de Bellary.

Cette différence entre arbres gélivés et arbres témoins à Vierzon, n'est toutefois vraie qu'au cœur, et dans l'autre sens; dans les autres positions (duramen et aubier) ils ne diffèrent pas significativement, comme le montre le tableau VIII.

\section{Retrait radial des arbres de 1988}

Le coefficient de variation résiduel reste très élevé $(54 \%$ pour Bellary, $30 \%$ pour Bitche, $13 \%$ pour Vierzon) : les facteurs étudiés décrivent assez mal la variabilité du retrait radial à Bellary et à Bitche.

Les résultats, présentés dans le tableau IX, sont identiques pour Bellary et Bitche : il n'apparaît pas de différence entre arbres gélivés et arbres témoins; pour Vierzon le résultat est identique à celui du retrait tangentiel : le retrait radial est supérieur pour les arbres restés indemnes.

Cette différence de retrait entre arbres gélivés et arbres témoins à Vierzon, se manifeste dans le "cœur" comme pour le retrait tangentiel (du grand côté seulement) mais aussi dans le duramen, comme le montre le tableau $X$.

À Vierzon, outre cet effet «position» très fort, le petit et le grand côté des arbres gélivés présentent une différence significative, comme le montre le tableau XI. 
Tableau VIII. Interaction gélivure-position à Vierzon sur le retrait tangentiel (en \%).

\begin{tabular}{|c|c|c|c|c|}
\hline & $\begin{array}{c}\text { Cceur } \\
\text { gélivés témoins }\end{array}$ & $\begin{array}{l}\text { Duramen } \\
\text { gélivés témoins }\end{array}$ & $\begin{array}{l}\text { Aubier } \\
\text { gélivés témoins }\end{array}$ & \\
\hline Vierzon & $10,05>8,98$ & $8,96=8,79$ & $5,36=5,38$ & *** \\
\hline
\end{tabular}

Tableau IX. Retraits radiaux des chênes de 1988 (en \%).

\begin{tabular}{llllll}
\hline & $\begin{array}{c}\text { Moyenne } \\
\text { générale }\end{array}$ & $\begin{array}{c}\text { Arbres } \\
\text { gélivés }\end{array}$ & $\begin{array}{c}\text { Arbres } \\
\text { témoins }\end{array}$ \\
\hline Bellary & 2,53 & $2,55=$ & 2,50 & NS \\
Bitche & 3,07 & 3,00 & $=$ & 3,15 & NS \\
Vierzon & 3,51 & $3,35<$ & $<, 68$ & \\
\hline
\end{tabular}

\section{Conclusion sur les retraits}

Aucune différence entre arbres gélivés et non-gélivés sur le retrait longitudinal n'a été observée, sans que l'on sache si la méthode de mesure de cette variable est assez précise pour faire apparaître une éventuelle différence.

D'une manière générale : le retrait tangentiel des arbres gélivés est supérieur ou égal à celui des arbres témoins, sauf à

Tableau X. Interaction gélivure-position à Vierzon sur le retrait radial (en \%).

\begin{tabular}{llcccc} 
& \multicolumn{2}{c}{ Cœur } & \multicolumn{2}{c}{ Duramen } & \multicolumn{2}{c}{ Aubier } \\
& témoins & gélivés & témoins & gélivés & témoins gélivés \\
\hline Vierzon & $4,42>3,77$ & $3,96>3,67$ & $2,66=2,60$ & \\
\hline
\end{tabular}

\section{Anisotropie de retrait}

L'étude de l'anisotropie de retrait, définie comme le rapport du retrait tangentiel au retrait radial, ne fait apparaître dans aucune des trois forêts de 1988 (tableau XII) de différence significative entre les arbres gélivés et les arbres témoins.

Les "anomalies" des résultats concernant les retraits (tableaux VIII et $X$ ) se retrouvent pour l'anisotropie qui présente une forte interaction des facteurs "gélivure» et «position», et une différence significative au seuil de $1 \%$ entre arbres gélivés et témoins, dans un sens opposé selon que l'on considère l'aubier ou le "cœur".
Tableau XI. Interaction gélivure-oposition à Vierzon sur le retrait radial (en \%).

Témoins
grand petit $\begin{gathered}\text { Gélivés } \\ \text { petit grand }\end{gathered}$

Vierzon (chêne pédonculé), où, au cœur des arbres témoins, il est très supérieur à celui des gélivés; le retrait radial des arbres gélivés est égal à celui des arbres témoins, sauf, de nouveau, à Vierzon, où il est supérieur, au cœur et dans le duramen des arbres témoins, à celui des gélivés. 
Tableau XII. Anisotropie de retrait des chênes de 1988 (sans unité).

\begin{tabular}{lllll}
\hline & $\begin{array}{c}\text { Moyenne } \\
\text { générale }\end{array}$ & $\begin{array}{c}\text { Arbres } \\
\text { gélivés }\end{array}$ & $\begin{array}{c}\text { Arbres } \\
\text { témoins }\end{array}$ \\
\hline Bellary & 2,86 & $2,87=2,86$ & NS \\
Bitche & 3,10 & $3,13=3,06$ & NS \\
Vierzon & 2,60 & $2,63=2,57$ & NS \\
\hline
\end{tabular}

La différence de retrait tangentiel entre les deux populations est parfois significative et plus grande que la différence de retrait radial. Ceci permet de supposer que la sensibilité au gel est en partie explicable par un retrait tangentiel plus fort. L'importance donnée par Kübler (1983) au retrait au gel est renforcée par nos résultats, bien que ce qui a été mesuré ici ne soit pas la rétractibilité au gel. tif pour l'angle de fil du bois. Ce résultat est d'autant plus fort que, dans le choix des arbres, ceux qui présentaient un fibre torse apparente avaient été systématiquement éliminés; cela explique que les angles mesurés soient si faibles.

L'interaction gélivure-zone est forte : les différences entre gélivés et témoins ne sont significatives que pour les deux zones extérieures. Ce résultat est intéressant puisque ce sont ces zones extérieures, qui présentent en outre un angle nettement supérieur à la moyenne, qui sont les premières soumises au froid.

\section{Arbres 1988}

Les résultats pour les chênes de 1988 sont voisins de ceux de 1987, pour le chêne sessile comme pour le pédonculé. Les angles moyens sont un peu plus forts,

Tableau XIII. Interaction gélivure-position à Vierzon sur l'anisotropie de retrait (sans unité).

\begin{tabular}{|c|c|c|c|c|c|}
\hline & $\begin{array}{c}A u \\
\text { témoins }\end{array}$ & $\begin{array}{l}\text { ubier } \\
\text { gélivés }\end{array}$ & $\begin{array}{c}\text { Duramen } \\
\text { gélivés témoins }\end{array}$ & \begin{tabular}{l}
\multicolumn{2}{c}{ Cœur } \\
gélivés témoins
\end{tabular} & \\
\hline Vierzon & $4,17>$ & 3,62 & $2,45=2,31$ & $1,80>1,24$ & $* *$ \\
\hline
\end{tabular}

\section{Angle de fil du bois et sensibité à la gélivure}

\section{Arbres 1987}

II faut tout d'abord remarquer que les facteurs étudiés n'expliquent qu'une faible partie de la variabilité puisque le coefficient de variation résiduel est d'environ $60 \%$. Ceci tient, entre autres, aux erreurs de précision signalées au paragraphe $\mathrm{Ma}$ tériels et méthodes. Sous cette réserve, le facteur "gélivure" a un effet très significa-
Tableau XIV. Angle du fil du bois des chênes de $1987\left(\mathrm{en}^{\circ}\right)$.

Moyenne Gélivés Témoins

\begin{tabular}{|c|c|c|c|c|c|}
\hline $\begin{array}{l}\text { Moyenne } \\
\text { arbres } 1987\end{array}$ & 1,14 & 1,34 & $>$ & 0,94 & $* * *$ \\
\hline Zone 9 & 1,71 & 2,14 & $>$ & 1,27 & $* \star \star *$ \\
\hline $\begin{array}{l}\text { Zone } 10 \\
\text { (aubier) }\end{array}$ & 1,79 & 2,24 & $>$ & 1,33 & $* * *$ \\
\hline
\end{tabular}


Tableau XV. Angle du fil du bois des chênes de $1988\left(\mathrm{en}^{\circ}\right)$.

Moyenne Gélivés Témoins

\begin{tabular}{llll}
\hline $\begin{array}{l}\text { Chênes } \\
\text { sessiles 1988 }\end{array}$ & 1,46 & $1,65>1,27$ \\
$\begin{array}{l}\text { Chênes } \\
\text { pédonculés } 1988\end{array}$ & 1,91 & $2,27>1,55 \quad * * *$ \\
\end{tabular}

mais la différence entre arbres gélivés et témoins est toujours dans le même sens. L'interaction gélivure-zone n'est pas significative. Comme pour les arbres de 1987 , les zones extérieures présentent un angle du fil du bois plus élevé que les zones proches de la moelle.

\section{Conclusion sur l'angle de fil du bois}

La comparaison des arbres gélivés et témoins montre que l'angle de fil du bois est toujours plus grand pour les premiers, au moins dans les zones externes. Ceci est à rapprocher du travail d'Archer mentionné dans Ferrand (1982) : "à la périphérie de la tige, à contraintes égales relativement au fil du bois, quand l'angle du fil augmente, la tension axiale diminue et la compression «tangentielle» (mesurée perpendiculairement à l'axe du tronc) diminue et peut même devenir une tension, ce qui augmente les risques de rupture puisque la gélivure résulte d'une tension tangentielle". Cela vient étayer les préventions qu'a généralement le sylviculteur face au défaut de fibre torse.

\section{Humidité hivernales des tiges et sensibilité à la gélivure}

Les résultats sont très variables d'une forêt à l'autre et d'un mois à l'autre, mais on peut relever que les arbres gélivés ont toujours une humidité significativement supé- rieure aux arbres témoins (au seuil de $0,1 \%$ ), comme le montre le tableau XVI. La discrimination gélivé-non gélivé apparaît ici valable pour toutes les forêts étudiées, ce qui n'a été le cas, il faut le souligner, pour aucun des autres caractères étudiés.

\section{Conclusion}

L'humidité constatée dans les tiges de chênes en hiver est située entre $60 \%$ et la saturation intégrale. C'est précisément le domaine d'humidité où l'expansion au gel (augmentation de volume sous l'effet de la prise en glace de l'eau contenue dans les vaisseaux) compense plus ou moins, selon le taux de remplissage des vaisseaux, le retrait au gel (Kübler, 1983; Cinotti, 1987). L'humidité hivernale des tiges pourrait ainsi être un facteur prédisposant à la gélivure.

\section{Largeurs de cernes et sensibilité à la gélivure}

\section{Arbres de 1987}

Sur la largeur moyenne de cernes sur l'ensemble du rayon calculée par division de

Tableau XVI. Humidités du bois des chênes de 1988 (en \%).

\begin{tabular}{|c|c|c|c|c|c|}
\hline \multirow[b]{2}{*}{ Bellary } & \multirow[t]{2}{*}{ Moyenne } & \multicolumn{2}{|c|}{ Gélivés } & \multicolumn{2}{|l|}{ Témoins } \\
\hline & & & & & \\
\hline janvier & 86,5 & 89,0 & $>$ & $84,0 *$ & *** \\
\hline février & 83,1 & 85,5 & $>$ & $80,8 *$ & **** \\
\hline mars & 81,0 & 83,6 & $>$ & $78,3 *$ & "*** \\
\hline \multicolumn{6}{|l|}{ Bitche } \\
\hline mars & 87,0 & 89,0 & $>$ & 85,0 * & **** \\
\hline \multicolumn{6}{|l|}{ Vierzon } \\
\hline février & 94,2 & 94,9 & $>$ & 93,4 * & * \\
\hline mars & 95,1 & 96,6 & $>$ & $93,6 *$ & ** \\
\hline
\end{tabular}


la circonférence par le nombre de cernes et par $2 \pi$, la différence entre arbres 1987 gélivés et non-gélivés était significative au seuil de $5 \%(1,76 \mathrm{~mm}$ pour les gélivés contre 1,58 pour les non-gélivés). Ce résultat est confirmé par l'analyse d'images effectuée sur la troisième zone en partant de la mcelle de sept de nos 17 couples de 1987 : la largeur moyenne dans cette zone des cernes des arbres gélivés $(1,86 \mathrm{~mm})$ est très significativement supérieure (au seuil de $0,1 \%$ ) à celle des arbres témoins $(1,42 \mathrm{~mm})$.

Une deuxième confirmation a été apportée lors de l'étude menée à la station de recherches sur la qualité des bois par Haag (1989) sur une base d'échantillons quasiment identique mais sur la totalité du rayon (et non plus sur une seule zone) : la largeur de cerne des arbres gélivés $(1,55$ $\mathrm{mm}$ ) diffère significativement (au seuil de $0,1 \%)$ de celles des arbres témoins $(1,34$ $\mathrm{mm})$.

\section{Arbres de 1988}

Pour chaque couple, les arbres gélivés et non-gélivés ont été choisis de diamètres semblables, et la largeur moyenne de cernes sur l'ensemble du rayon était identique dans les deux groupes comme cela a été vérifié (tableau I et II). Or, il apparaît une différence significative dans deux des trois forêts (Bellary et Vierzon) pour la largeur de cerne mesurée position par position (tableau XVII). Cette différence y est d'ailleurs de signe opposé.
Cette contradiction apparente se résoud par le rappel de la définition de la zone "cœur", puisque cette zone (limitée aux 15 premiers cernes en partant de la moelle) bénéficie d'un poids démesuré dans notre analyse de la largeur de cernes mesurée position par position.

Ceci apparaît de façon très nette à Bellary où l'interaction gélivure-position est très forte (tableau XVIII) et où la seule position dans laquelle existe effectivement une différence significative entre arbres gélivés et témoins est le "cœur".

En ce qui concerne Vierzon, il faut admettre que le choix d'arbres de diamètre égal a masqué un biais sur l'âge (tableau II). II s'ensuit une différence significative entre arbres gélivés et témoins sur la largeur moyenne de cernes, que l'interaction gélivure-position confirme position par position (tableau XIX).

\section{Conclusion sur les largeurs de cernes}

L'étude des largeurs de cernes position par position apporte, malgré l'apparente

Tableau XVII. Largeurs de cernes des chênes de 1988 (en mm).

\begin{tabular}{lllll}
\hline & Moyenne Gélivés & \multicolumn{2}{c}{ Témoins } \\
\hline Bellary 1,90 & $1,82<1,97 \quad * * *$ \\
Bitche 1,40 & $1,43=1,37 \quad$ NS \\
Vierzon 1,34 & $1,53>1,15 \quad * *$ \\
\hline
\end{tabular}

Tableau XVIII. Interaction gélivure-position sur la largeur de cernes à Bellary (en mm).

\begin{tabular}{llcccc} 
& \multicolumn{2}{c}{ Cour } & \multicolumn{2}{c}{ Duramen } & \multicolumn{2}{c}{ Aubier } \\
& témoins & gélivés & témoins & gélivés & témoins gélivés \\
\hline Bellary & 2,37 & $>2,08$ & $1,99=1,96$ & $1,55=1,43 \quad * *$ \\
\hline
\end{tabular}


contradiction avec la largeur moyenne de cernes sur l'ensemble du rayon (ou le biais noté pour les chênes pédonculés de Vierzon), la confirmation que les arbres gélivés et témoins peuvent différer dans certaines zones du rayon de l'arbre. On ne pourra guère tirer d'autres conclusions. Toute étude sur le sujet devrait s'attacher à étudier aussi la régularité de la largeur de cerne sur tout le rayon.

\section{Caractéristique anatomiques et sensibilité à la gélivure}

\section{Arbres de 1987}

\section{Pourcentage de bois initial} et sensibilité à la gélivure

Dans les deux études présentes au paragraphe Matériels et Méthodes, le pourcentage de bois initial des cernes des arbres gélivés est apparu très significativement inférieur (au seuil de $0,1 \%$ ) à celui des arbres témoins, mais cette différence était inférieure à l'erreur de répétabilité (effet cerne plus effet mesure) qui est de $4 \%$.

(Dans ce tableau et dans ceux qui suivent, la différence de résultats entre Haag et nous-même vient de ce que, bien que travaillant sur les mêmes arbres, nous n'avons pas étudié les mêmes zones du rayon).

Cette tendance concernant la texture peut cependant être reliée à celle de la largeur de cerne, puisqu'il a été observé (Polge et Keller, 1973) que le pourcentage de bois initial du chêne diminue quand la largeur de cerne augmente. "L'importance des phénomènes de retrait et notamment de retrait tangentiel est variable selon la texture du bois. Dans le cas du chêne la texture est plus forte, et par conséquent le bois plus fissile, chez les arbres à accroissements larges que chez ceux qui ont crû lentement» notait Rol (1953). Malheureusement nous ne pouvons pas établir de corrélations entre la texture et le retrait tangentiel de nos arbres, les dispositifs de mesures de ces données n'ayant pas été les mêmes. Enfin, la comparaison des largeurs de bois initial entre arbres gélivés et arbres témoins, en ne faisant pas apparaître de différence significative entre eux (Haag, 1989), confirme que la différence de texture est due à l'importance de la largeur du bois final, donc de largeur de cerne.

Tableau XIX. Interaction gélivure-position sur la largeur de cernes à Vierzon (en mm).

\begin{tabular}{llllllll} 
& \multicolumn{2}{c}{ Cour } & \multicolumn{2}{c}{ Duramen } & \multicolumn{2}{c}{ Aubier } \\
& gélivés & témoins & gélivés & témoins & gélivés & témoins \\
\hline Vierzon & 2,06 & $>1,47$ & $1,21>0,95$ & $1,32>1,02$ & $* * *$ \\
\hline
\end{tabular}

Tableau XX. Pourcentage de bois initial des chênes de 1987 (en \%).

\begin{tabular}{lllll}
\hline & Gélivés & \multicolumn{2}{c}{ Témoins } \\
\hline Cinotti & 23 & $<$ & 28 & \\
Haag & 34 & $<$ & 37 & $* \star *$ \\
\hline
\end{tabular}

\section{Surface des vaisseaux du bois initial} et sensibilité à la gélivure

II s'agit sans doute du résultat le plus important de l'analyse d'images: la surface individuelle moyenne des vaisseaux du bois initial des arbres gélivés est significativement supérieure à celle des arbres témoins. 
Tableau XXI. Surface moyenne des gros vaisseaux du bois initial des chênes de 1987 (en $\mu \mathrm{m}^{2}$ ).

\begin{tabular}{llll}
\hline & Gélivés & Témoins \\
\hline Cinotti & $31000>28000 \quad * \star$ \\
Haag & $45800>43400 \quad * \star *$ \\
\hline
\end{tabular}

Malgré la faible taille de la population étudiée, ce résultat est d'autant plus important qu'il vient corroborer ce qu'avait trouvé Savill (1986) qui étudiait, sur grumes abattues, la sensibilité des chênes anglais à toutes sortes de fentes (roulure, cadranure, fente de retrait). II ouvre des pers-pectives d'étude du rôle que peuvent jouer les vaisseaux du bois initial dans la distribution et les transferts d'eau dans la tige. II serait aussi très intéressant de poursuivre l'étude des relations entre l'anatomie et la mécanique de l'arbre du séchage, du retrait au gel ou d'autres sollicitations.

Pourcentage (en surface) de vaisseaux dans le bois initial et sensibilité à la gélivure

L'analyse d'images n'a pas permis de tirer des résultats statistiquement significatifs. $\mathrm{Ce}$ point mériterait peut-être d'être revu lors d'études anatomiques plus poussées.

Tableau XXII. Pourcentage des gros vaisseaux dans le bois initial des chênes de 1987 (en \%).

\begin{tabular}{lllll}
\hline & Gélivés & \multicolumn{3}{c}{ Témoins } \\
\hline Cinotti & $30=$ & 32 & NS \\
Haag & $46=$ & 46 & NS \\
\hline
\end{tabular}

Pourcentage (en surface) de fibres et sensibilité à la gélivure

L'analyse de variance sur cette variable ne fait apparaître aucune différence significative entre les deux populations gélivésnon gélivés. Il faut cependant se garder de conclure trop rapidement car la répétabilité de la mesure du pourcentage de fibres donne une erreur de 2,5\% (Haag, 1989), supérieure à la différence entre les deux populations.

\section{Arbres de 1988 : Étude du nombre des gros rayons ligneux}

Cette analyse a fait apparaître, pour deux des trois forêts, une différence très significative entre arbres gélivés et arbres témoins. Il n'y a pas d'effet opposition, ni d'interaction opposition-gélivure.

\section{Conclusion sur l'anatomie}

La surface des vaisseaux du bois initial est plus forte pour les arbres gélivés, ce qui confirme les travaux de Savill (1987) sur la sensibilité aux fentes des arbres à gros vaisseaux. La texture, la largeur de cerne et le nombre de rayons ligneux par unité de longueur sur une corde tangentielle seraient également plus élevés pour les arbres gélivés. Cela n'est pas vraiment étonnant compte tenu de la relation généralement admise entre largeur de cerne,

Tableau XXIII. Pourcentage de fibres des chênes de 1987 (en \%).

\begin{tabular}{llllll}
\hline & Gélivés & \multicolumn{2}{c}{ Témoins } \\
\hline Cinotti & 44 & $=$ & 45 & NS \\
Haag & 29 & $>$ & 27 & $*$ \\
\hline
\end{tabular}


Tableau XXIV. Nombre de rayons ligneux sur une corde de $3,5 \mathrm{~cm}$.

\begin{tabular}{lllll}
\hline & Gélivés & \multicolumn{3}{c}{ Témoins } \\
\hline Moyenne & 15,45 & $>$ & 14,57 & $* * *$ \\
Bellary & 15,27 & $>$ & 14,37 & $* *$ \\
Bitche & 15,76 & $>$ & 14,19 & $* * *$ \\
Vierzon & 15,27 & $=$ & 15,22 & NS \\
\hline
\end{tabular}

densité et texture. Le résultat est plus nouveau pour les rayon ligneux.

\section{CONCLUSION}

La comparaison d'arbres gélivés et nongélivés appariés par leur diamètre, leur forme extérieure, leur espèce et leur proximité sur le terrain montre que plusieurs caractéristiques anatomiques et physiques peuvent différer significativement d'une population à l'autre, ceci malgré la grande variabilité de ces propriétés chez les chênes. Ainsi, l'angle de fil du bois, l'infradensité, I'humidité hivernale, le retrait tangentiel, et dans une moindre mesure le retrait radial se sont avérés généralement plus élevés pour les arbres gélivés que pour les arbres demeurés indemnes.

Ces résultats sont importants dans la mesure où ils confirment ce que pensaient Kübler (1983), et Denne et Henman (1984) : tout ce qui augmente les contraintes que subit le tronc d'un arbre joue un rôle de facteur prédisposant.

De même, quelques caractères anatomiques sont apparus plus élevés pour les arbres gélivés : la largeur moyenne de cerne, la texture, la surface des vaisseaux du bois initial, le nombre de rayons ligneux par unité de longueur.

Certes, aucun de ces paramètres ne permet à lui seul de discriminer nettement arbres sensibles et arbres non sensibles à la gélivure; nous touchons là une des limites de notre travail.

Ceci est dû en partie au fait que le choix des individus des deux populations (appariement en circonférence pour éliminer les effets de milieu et de forme dans l'analyse) n'a pas évité une importante interaction couple-gélivure dans l'échantillon étudié. Cette interaction traduit probablement à la fois la très grande variabilité des propriétés $\mathrm{du}$ bois, et la complexité des relations entre ces propriétés et la sensibilité à la gélivure.

De plus, la plupart des paramètres individuels sont étroitement liés aux conditions stationnelles dont les arbres ont bénéficié ou pâti pendant leur croissance.

Or, ces relations sont encore mal connues. Faute d'étude sur ce point, nous ne savons pas, par exemple, si l'humidité est sous contrôle stationnel par le biais des phénomènes de capillarité qui pourraient expliquer tout ou partie des différences d'humidité hivernale, ou si elle est une caractéristique individuelle.

Cette étude sur les relations entre les propriétés de base du bois des chênes et leur sensibilité à la gélivure prendra tout son sens lorsque de nouveaux résultats sur les liaisons sylviculture - propriétés de base du bois de chênes et sur l'héritabilité des caractéristiques principales du plan ligneux (surface des vaisseaux, pourcentage de fibres, fraction volumique en rayons ligneux) auront été apportés par les équipes qui travaillent sur l'anatomie. II serait très intéressant surtout de poursuivre l'étude des relations entre l'anatomie et la mécanique de l'arbre lors du séchage, du retrait au gel ou d'autres sollicitations. II y a beaucoup à faire pour étudier les relations entre l'initiation de la rupture et la disposition ou les caractéristiques de certains éléments anatomiques tels que les rayons ligneux. 
Notre travail a dégagé des pistes assez intéressantes, mais nous ne sommes pas en mesure d'apporter des critères de décision aux améliorateurs pour le cas où ils s'intéresseraient au chêne, même si les caractéristiques étudiées sont mesurables, à l'exception de l'angle de fil et du nombre des rayons ligneux, sur carottes de sondage.

\section{REMERCIEMENTS}

Cette étude a été réalisée grâce aux financements du ministère de l'Agriculture et de la Forêt, direction scientifique de l'École nationale du Génie Rural, des Eaux et des Forêts et direction de l'Espace Rural et de la Forêt.

\section{RÉFÉRENCES}

Arbois de Jubainville (d') A, Vesque $J$ (1878) Les maladies des plantes cultivées, des arbres forestiers et fruitiers. Rothschild, Paris, 52-56

Cinotti B (1987) Influence de la structure du bois des chênes (Quercus Robur $L$ et $Q$ petraea Liebl) sur leur sensibilité à la gélivure. DEA sciences du bois, Nancy, INRA

Cinotti $B$ (1989) La gélivure des chênes : front de gel source de contraintes internes, incidence des propriétés anatomiques et mécanophysiques. Nancy, Thèse de doctorat INPL en sciences du bois

Cinotti B (1990) Winter moisture-content and frost-crack occurrence in oak trees (Quercus petraea Liebl et $Q$ robur L). IUFRO symposium international sur la physiologie des arbres forestiers, Nancy, septembre 1988. Ann Sci For 46 (Suppl) 614s-616s

Denne P, Henman S (1984) Control of wood quality in British oaks. Report on forest research. Forestry Commission, 59

Ferrand JC (1982) Étude des contraintes de croissance. Deuxième partie : variabilité en forêt des contraintes de croissance du hêtre. Ann Sci For 39 (3), 187-218

Haag H (1989) Étude de l'influence de la structure anatomique du bois sur la sensibilité du chêne rouvre (Quercus petraea Liebl) à la gélivure. Mirecourt, Rapport de stage de BTS, option Prod Forestière

Institut pour le Développement Forestier (IDF) (1988) La gélivure des chênes. Rapport de préétude

Kübler $H$ (1983) Mechanism of frost cracks formation in trees. A review and synthesis. For Sci 29, 559-568, traduit en français par Bruno Cinotti

Miller $P$ (1987) La gélivure des chênes pédonculé et sessile dans le centre de la France. Aspects descriptif, stationnel, technologique et sylvicole. Mémoire de $3^{\theta}$ année ENITEF, $70 \mathrm{p}$

Polge H, Keller R (1973) Qualité du bois et largeur d'accroissement en forêt de Tronçais. Ann Sci For 30 (2) 91-125

Rol R (1953) Les gélivures. Bull Soc For Franche Comté, 642-651

Savill PS (1986) Anatomical characters in the wood of Oak (Quercus robur $L$ and $Q$ petraea Liebl) which predispose trees to shake. Commonw For Rev 65 (2), 109-116 